\title{
IMPLICAÇÕES ETNOCONSERVACIONISTAS QUANTO AO MANEJO INFORMAL DO MARISCO Anomalocardia fleXUOSa (LINNAEUS, 1767) POR MARISQUEIRAS
}

\author{
Ethnoconservational implications regarding informal \\ shellfish management Anomalocardia flexuosa (Linnaeus, \\ $1767)$ by shellfish gathering women
}

\author{
Ivo Raposo Gonçalves Cidreira-Neto', Gilberto Gonçalves Rodrigues² \\ 1 Doutorando em Desenvolvimento e Meio Ambiente (PRODEMA) na Universidade Federal de Pernambuco. \\ Av. Professor Moraes Rego, 1235, Cidade Universitária, Recife-PE. E-mail: ivo.raposo@hotmail.com \\ ${ }^{2}$ Professor do Departamento de Zoologia na Universidade Federal de Pernambuco. Av. Professor Moraes \\ Rego, 1235, Cidade Universitária, Recife-PE. E-mail: gilbertorodrigues.ufpe@gmail.com
}

\begin{abstract}
RESUMO
A mariscagem pode ser compreendida como a pesca artesanal de moluscos, onde o seu manejo pode ser realizado de forma manual ou com o auxílio de apetrechos de pesca, sendo o principal para a região Nordeste a exploração e comercialização do marisco (Anomalocardia flexuosa). O objetivo deste trabalho foi analisar a prática do manejo informal do marisco, coletado manualmente por marisqueiras no litoral nordeste do Brasil. O local de estudo foi a Reserva Extrativista Acaú-Goiana, situada entre os estados de Pernambuco e Paraíba. A pesquisa foi realizada durante os anos de 2017 e 2018, constando com imersões e visitas aleatórias nas comunidades de Acaú e Carne de Vaca, usando como base a observação participante. A partir da separação em tamanhos miúdos e graúdos, adotados pelas marisqueiras, foram realizadas medições para a altura, comprimento e largura das conchas. Ao todo foram acompanhadas a catação e separação dos mariscos por 11 marisqueiras, totalizando 1.793 mariscos, sendo 685 miúdos (desprezados) e 1.108 graúdos. O tamanho de intersecção entre catar ou rejeitar o marisco ocorre com os tamanhos de 18 a $20 \mathrm{~mm}$ para o comprimento total, o que é sustentável do ponto de vista biológico. Essa ação resulta na diminuição do processo de sobrepesca, pois os mariscos catados já estão sexualmente adultos, ou seja, já passaram pelo processo de reprodução, e os desprezados podem se reproduzir.
\end{abstract}

Palavras-chave: unidade de conservação, comunidade pesqueira, conservação, pesca artesanal.

Recebido em: 05/04/2019

Aprovado em: 07/08/2019

Publicado online em: 10./11/2019 


\section{ABSTRACT}

The mollusk gathering activity can be understood as the artisanal fishing of molluscs, where their management can be carried out manually or with the aid of fishing gear being the main one for the Northeast region the exploitation and commercialization of shellfish (Anomalocardia flexuosa). The objective of this work was to analyze the practice of the informal management of shellfish, manually collected by mollusk gathering in the northeast coast of Brazil. The study site was the Acaú-Goiana Extractive Reserve, located between the states of Pernambuco and Paraíba. The research was carried out during the years 2017 and 2018, consisting of immersions and random visits in the communities of Acaú and Carne de Vaca, based on participant observation. From the separation in the small and large sizes, adopted by the mollusk gathering, measurements were made for the height, length and width of the shells. A total of 11 shellfish were culled and separated from the mollusk gathering, totaling 1793 shellfish, totalizing of 1,793 selfish, 685 small (discarded) and 1108 gross. The size of intersection between picking or discarding shellfish occurs with sizes from 18 to $20 \mathrm{~mm}$ for the overall length, which is biologically sustainable. This action results in a reduction of the overfishing process, since the seafood caught is already sexually adult, that is, they have already passed through the process of reproduction, and the despised can

Keywords: conservation unit, fishing community, conservation, artisanal fishing.

\section{INTRODUÇÃO}

A pesca artesanal é uma das mais antigas atividades realizadas no mundo, sendo caracterizada como uma pequena produção mercantil, utilizando peixes, crustáceos e moluscos (Diegues, 1983). Entre os moluscos, os bivalves que mais apresentam expressividade na pesca artesanal brasileira, bem como na sua ampla comercialização, são: (i) Anomalocardia flexuosa (Linnaeus, 1767), conhecido popularmente como marisco; (ii) Mytella spp., conhecida como sururu; e (iii) Crassostrea spp., conhecida como ostra.

A mariscagem, que compreende a catação dos mariscos (A. flexuosa), é uma atividade que traz representatividade feminina, além de agregar complexas relações e entendimentos quanto ao território pesqueiro, construídos a partir da prática e transmissão através da oralidade (Bezerril, 2012; Caetano, 2015). O conhecimento das marisqueiras sobre os fatores que atuam no estuário é diverso, entendendo de temas como variação de temperatura, pH e salinidade, assim como de distribuição, biologia e ecologia dos organismos coletados (Nishida; Nordi \& Alves, 2004; Souto \& Martins, 2009). Esse conjunto de conhecimentos e crenças é conhecido como Conhecimento Ecológico Local (CEL), que parte das construções sociais passando através das gerações (Berkes, 1999).

A pesca do marisco ocorre nas áreas de praia e manguezais, principalmente nos momentos de maré baixa, o que possibilita o aparecimento das croas (banco areno/lodoso) utilizadas para coleta, sendo realizada em grupos sociais ou individuais. Existem duas formas principais de pesca: a manual, onde os mariscos são coletados através da extração direta no sedimento, e a com o auxílio de apetrechos artesanais, como puçá, gadanho e jereré, que aumentam a capacidade de coleta (Silva \& Martins, 2017). A pesca manual do marisco possibilita que as pescadoras optem principalmente pelos maiores tamanhos, pela premissa de que estes vão apresentar um maior rendimento da carne. 
Dessa forma, o manejo informal do marisco, realizado a partir da catação e beneficiamento manual, agrega valor ao produto final, valorizando o trabalho artesanal realizado pelas pescadoras.

É necessário o incentivo das ações e práticas sustentáveis provenientes das comunidades tradicionais/populações locais, agregando o conhecimento científico e o conhecimento local para a construção de uma nova conservação, que vai além da singela romantização dos povos e populações tradicionais (Diegues, 2000). A etnoconservação vem como "uma possibilidade em potencial para a proteção dos recursos naturais" (Pereira \& Diegues, 2010, p. 47).

O surgimento da etnoconservação ganha ainda mais força no contexto das unidades de conservação de uso sustentável, possibilitando a inclusão de novas formas de gestão, construídas de forma sustentável e harmônica, em relação ao meio ambiente e as comunidades ali presentes (Barbosa \& Aguiar, 2018). Nesse contexto, relacionando com a pesca artesanal do marisco, tem-se a necessidade de introduzir estudos etnoconservacionistas nas Reservas Extrativistas Marinhas, visto que elas são responsáveis pela proteção do ecossistema e perpetuação da atividade pesqueira.

Dessa forma, o presente estudo teve como objetivo analisar a prática do manejo informal do marisco, coletado manualmente por marisqueiras no litoral Nordeste do Brasil.

\section{MATERIAL E MÉTODOS}

\section{Área de estudo}

A pesquisa foi realizada na Reserva Extrativista (RESEX) Acaú-Goiana (Figura 1), uma unidade de conservação (UC) de uso sustentável que está situada entre os estados de Pernambuco (litoral norte) e Paraíba (litoral sul), Nordeste do Brasil. Instituída em 2007, tornou-se um marco de conquista de luta das marisqueiras, que tomaram frente da criação da RESEX. A gestão da UC é realizada pelo Instituto Chico Mendes de Conservação da Biodiversidade (ICMBio), juntamente com o Conselho Gestor Deliberativo, contando com a participação de pescadores e pescadoras nas tomadas de decisão (Lima; Selva \& Rodrigues, 2016).

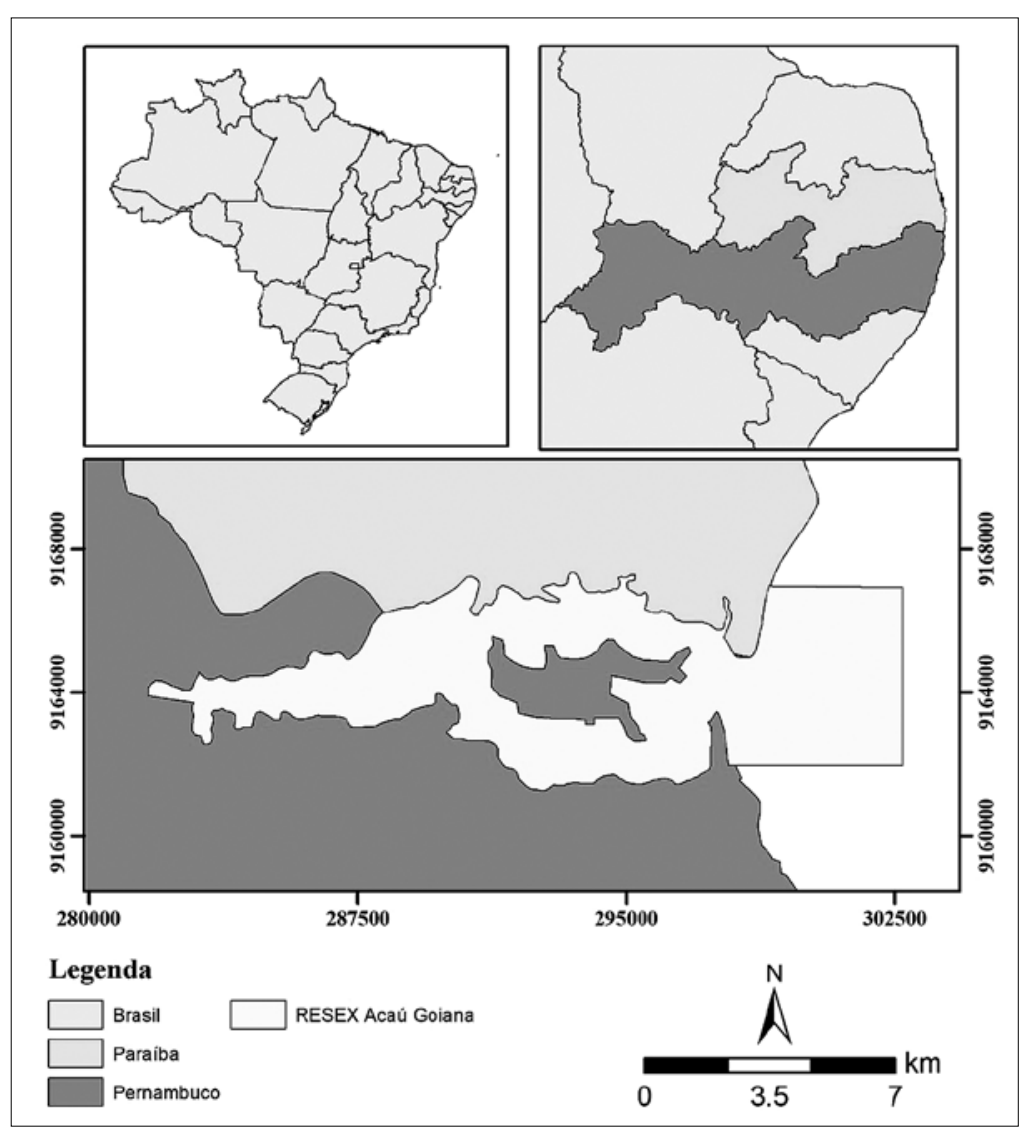

Figura 1 - Localização da Reserva Extrativista Acaú-Goiana 
Existem seis comunidades de pescadoras que são beneficiárias da RESEX, em que duas estão situadas no município de Pitimbu (Acaú) e Caaporã (Porto de Congaçari), no estado da Paraíba, e quatro no município de Goiana (Carne de Vaca, Povoação São Lourenço, Tejucupapo e Balde do Rio), em Pernambuco. No centro da UC estão situados os viveiros de camarão, que datam de um período anterior ao da implementação da RESEX, ocasionando diversos conflitos socioambientais, desde a poluição dos rios até confrontos com os pescadores, conforme relatado por eles mesmos.

A RESEX Acaú-Goiana consta com um Acordo de Gestão, publicado na Portaria $n^{o}$ 851, de 2017, contendo normativas internas para o uso dos recursos locais pelos beneficiários da unidade de conservação. Especificamente acerca da pesca do marisco, existe um tamanho mínimo para a coleta desse recurso, sendo de $15 \mathrm{~mm}$, evitando, assim, o efeito da sobrepesca.

\section{Coleta e análise de dados}

Por apresentarem a maior proximidade com as principais croas utilizadas para esse tipo específico de pesca, apenas as marisqueiras das comunidades de Carne de Vaca (PE) e Acaú (PB) participaram do estudo.

A pesquisa foi realizada com especialistas nativos, ou seja, pescadoras que são reconhecidas pelos comunitários como detentoras do conhecimento pesqueiro, tendo a pesca como a sua principal fonte de renda. Todas utilizam os recursos pesqueiros provenientes dos rios Megaó e Goiana, bem como o estuário formado pelo encontro desses dois rios com o mar, sendo a atividade de mariscagem (catação artesanal de marisco) a mais representativa.

Os dados foram coletados durante os anos de 2017 e 2018, a partir de imersões nas comunidades estudadas, sendo cinco dias seguidos em Acaú, contando com mais duas visitas esporádicas. Já em Carne de Vaca, foram realizadas duas imersões de quatro dias consecutivos, contanto também com uma visita esporádica.

A técnica utilizada foi a de observação participante (Gil, 2008), possibilitando que o pesquisador se aproprie de todas as etapas do evento observado, deixando de ser um expectador e participando ativamente de todos os processos. No caso do estudo, as marisqueiras foram acompanhadas durante o manejo do marisco, principalmente durante os momentos de pesca nas croas de coleta. Nesse momento, foi solicitado que as pescadoras separassem uma população de marisco, previamente coletado no mesmo estuário, nos tamanhos que consideravam aptos para a coleta, separando em miúdos (desprezados) e graúdos (hábil para pesca).

Em laboratório, os grupos passaram por medições da medida máxima do comprimento da concha (CC), largura da concha (LC) e a altura da concha (AC), com o auxílio de um paquímetro digital. Esse padrão segue o realizado por López-Rocha (2018).

Por questões éticas e legais, tem-se a aprovação pelo Comitê de Ética Humana da Universidade Federal de Pernambuco ( $\mathrm{n}^{\circ}$ 2.337.271), bem como pelo ICMBio, através do Sistema de Autorização e Informação em Biodiversidade (SISBIO n ${ }^{\circ}$ 58139-1).

\section{RESULTADOS E DISCUSSÃO}

Ao total, 11 marisqueiras auxiliaram durante o processo de pesca e separação dos mariscos, sendo oito moradoras de Carne de Vaca e três de Acaú. As croas utilizadas para a coleta foram a de Acaú (conhecida também como pontinhas), Taioba e Canoé (Figura 2). 


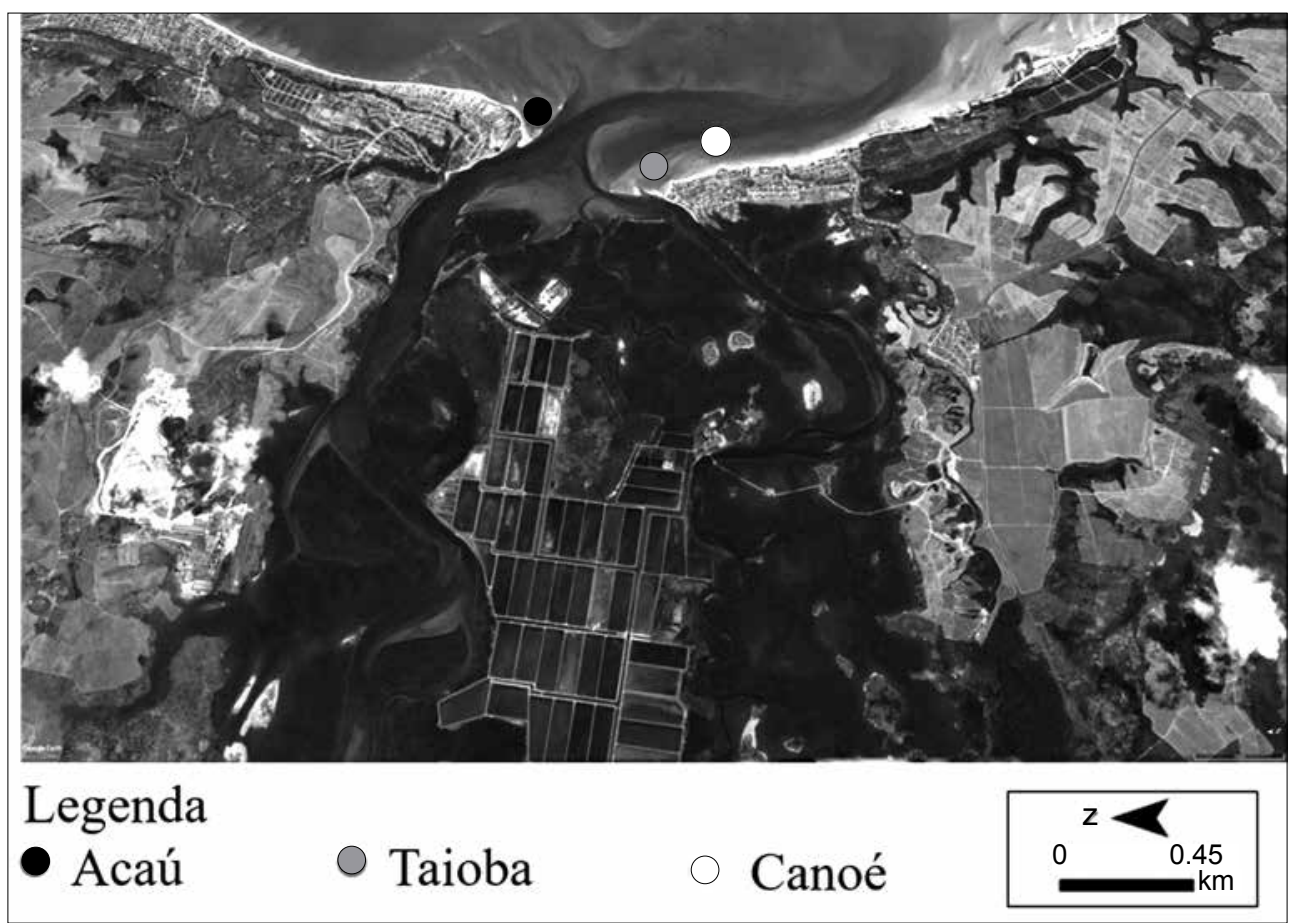

Figura 2 - Localização das croas utilizadas para a pesca do marisco

A escolha das comunidades de Carne de Vaca e Acaú foi devido à maior proximidade com os locais de pesca e por apresentar uma relevante representatividade da pesca manual do marisco. Já em relação as croas, essas são as principais utilizadas pelas marisqueiras que optam pela pesca sem apetrechos.

A média de idade das pescadoras que auxiliaram o estudo foi de 52 anos. Um fator importante quando se analisa a idade dessas marisqueiras é a média, o que pode inferir que a opção pela pesca manual, com separação dos tamanhos no próprio estuário, é algo adotado pelas mais experientes, que apresentam um sentimento de pertencimento ao local, respeitando o estuário. Segundo relato das próprias pescadoras, os mais novos (principalmente homens) preferem fazer a pesca com o auxílio de apetrechos, realizando a separação nas galeias (caixotes), onde são colocados os mariscos e agitados, para aqueles que apresentam tamanho pequeno possam cair no próprio estuário: "faz a seleção em uma galeia, mas como se galeia na maré, a maioria dos pequenos fica [...] tem lugar que eles vão galear no seco então os mariscos morrem" (relato de uma marisqueira). Além de uma pesca de maior impacto, essa prática pode ocasionar a morte dos indivíduos devido à quebra das conchas, além de uma separação não confiável.

Para realizar as medidas de CC, LC e AC, foram utilizados 1.793 mariscos, todos coletados nas mesmas croas utilizadas pelas marisqueiras. Com relação ao padrão dos tamanhos encontrados para essas medidas (Figura 3), obtiveram-se os seguintes intervalos mais expressivos, sendo: (i) AC - 16 a $18 \mathrm{~mm}$; (ii) LC - 11 a $13 \mathrm{~mm}$ e (iii) CC - 18 a $20 \mathrm{~mm}$.

Com relação à separação dos tamanhos realizada pelas marisqueiras, foram gerados os seguintes grupos: (i) miúdos, com 685 mariscos, e (ii) graúdos, com 1.108 mariscos (Tabela I). Dessa forma, o momento de intersecção entre catar ou rejeitar o marisco ocorre com os tamanhos acima de $10 \mathrm{~mm}$ para LC, $18 \mathrm{~mm}$ para CC e $14 \mathrm{~mm}$ para AC. A prática de separação manual dos mariscos coletados em tamanhos diferentes na hora da catação é uma atividade intrínseca, realizada com a finalidade de deixar os miúdos crescerem (Dias; Rosa 
\& Damasceno, 2007), para que eles possam reproduzir e não acabar com a principal fonte de renda das marisqueiras.

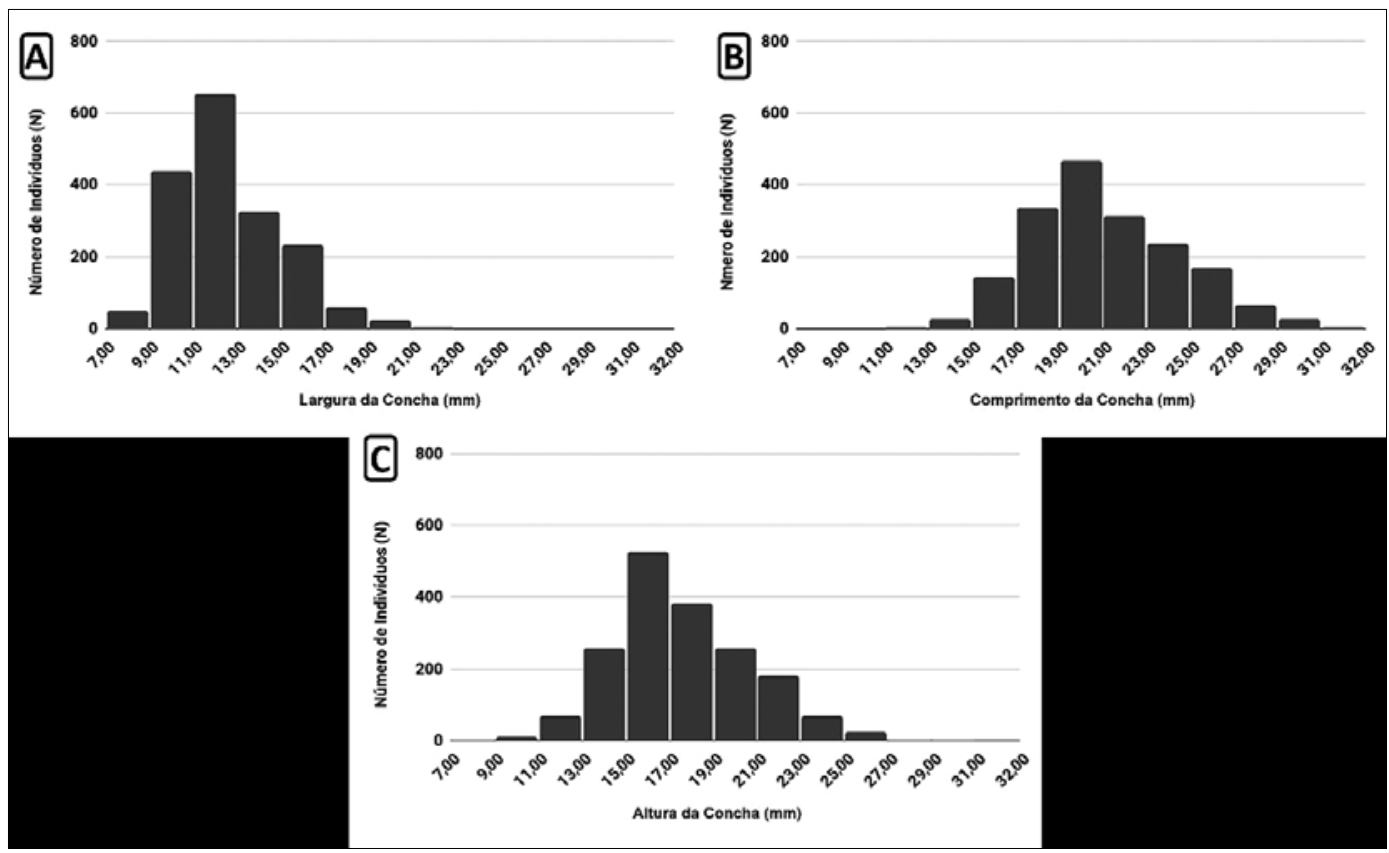

Figura 3 - Distribuição da frequência dos tamanhos de marisco (Anomalocardia flexuosa) coletados no estuário da Reserva Extrativista Acaú-Goiana

Nota: A. Frequência da Largura da Concha (CC). B. Frequência do Comprimento da Concha (CC). C. Frequência da Altura da Concha (AC).

Tabela 1 - Largura, comprimento e altura (média e desvio padrão) dos mariscos miúdos e graúdos classificados pelas marisqueiras da RESEX Acaú-Goiana, Nordeste do Brasil

\begin{tabular}{|c|c|c|c|c|c|c|c|c|}
\hline \multirow[b]{2}{*}{ Amostras } & \multicolumn{4}{|c|}{ Miúdos } & \multicolumn{4}{|c|}{ Graúdos } \\
\hline & $\mathrm{N}$ & LC & $\mathrm{CC}$ & $\mathrm{AC}$ & $\mathrm{N}$ & LC & $\mathrm{CC}$ & $\mathrm{AC}$ \\
\hline M1 & 97 & $\begin{array}{c}10,33 \\
( \pm 1,15)\end{array}$ & $\begin{array}{c}18,45 \\
( \pm 1,97)\end{array}$ & $\begin{array}{c}14,76 \\
( \pm 1,73)\end{array}$ & 100 & $\begin{array}{c}14,02 \\
( \pm 1,71)\end{array}$ & $\begin{array}{c}23,62 \\
( \pm 1,95)\end{array}$ & $\begin{array}{c}19,69 \\
( \pm 1,85)\end{array}$ \\
\hline M2 & 65 & $\begin{array}{c}11,20 \\
( \pm 1,39)\end{array}$ & $\begin{array}{c}19,61 \\
( \pm 2,15)\end{array}$ & $\begin{array}{c}15,76 \\
( \pm 2)\end{array}$ & 85 & $\begin{array}{c}14,66 \\
( \pm 1,95)\end{array}$ & $\begin{array}{c}24,42 \\
( \pm 2,72)\end{array}$ & $\begin{array}{c}20,48 \\
( \pm 2,77)\end{array}$ \\
\hline M3 & 100 & $\begin{array}{c}10,26 \\
( \pm 0,98)\end{array}$ & $\begin{array}{c}18,01 \\
( \pm 1,46)\end{array}$ & $14,52( \pm 1,49)$ & 59 & $\begin{array}{c}12,56 \\
( \pm 1,63)\end{array}$ & $\begin{array}{c}21,58 \\
( \pm 2,52)\end{array}$ & $\begin{array}{c}17,84 \\
( \pm 2,21)\end{array}$ \\
\hline M4 & 96 & $\begin{array}{c}9,52 \\
( \pm 1,14)\end{array}$ & $\begin{array}{c}16,62 \\
( \pm 1,88)\end{array}$ & $13,67( \pm 1,64)$ & 94 & $\begin{array}{c}13,52 \\
( \pm 1,51)\end{array}$ & $\begin{array}{c}22,46 \\
( \pm 2,05)\end{array}$ & $\begin{array}{c}19,13 \\
( \pm 1,99)\end{array}$ \\
\hline M5 & 25 & $\begin{array}{c}10,52 \\
( \pm 1,18)\end{array}$ & $\begin{array}{c}18,87 \\
( \pm 1,73)\end{array}$ & $14,98( \pm 1,56)$ & 108 & $\begin{array}{c}14,21 \\
( \pm 1,31)\end{array}$ & $\begin{array}{c}23,59 \\
( \pm 2,10)\end{array}$ & $\begin{array}{c}19,60 \\
( \pm 1,46)\end{array}$ \\
\hline M6 & 3 & $\begin{array}{c}8,90 \\
( \pm 0,62)\end{array}$ & $\begin{array}{c}16,80 \\
( \pm 1,83)\end{array}$ & $13,43( \pm 1,33)$ & 110 & $\begin{array}{c}15,28 \\
( \pm 3,31)\end{array}$ & $\begin{array}{c}24,41 \\
( \pm 3,80)\end{array}$ & $\begin{array}{c}20,57 \\
( \pm 3,53)\end{array}$ \\
\hline M7 & 36 & $\begin{array}{c}9,80 \\
( \pm 0,69)\end{array}$ & $\begin{array}{c}16,87 \\
( \pm 1,32)\end{array}$ & $13,59( \pm 1,06)$ & 126 & $\begin{array}{c}12,57 \\
( \pm 1,77)\end{array}$ & $\begin{array}{c}20,97 \\
( \pm 2,48)\end{array}$ & $\begin{array}{c}17,57 \\
( \pm 2,46)\end{array}$ \\
\hline M8 & 51 & $\begin{array}{c}9,84 \\
( \pm 1,94)\end{array}$ & $\begin{array}{c}16,75 \\
( \pm 2,09)\end{array}$ & $13,31( \pm 1,73)$ & 99 & $\begin{array}{c}14,35 \\
( \pm 1,76)\end{array}$ & $\begin{array}{c}23,84 \\
( \pm 2,22)\end{array}$ & $\begin{array}{c}19,99 \\
( \pm 2,31)\end{array}$ \\
\hline M9 & 68 & $\begin{array}{c}10,25 \\
( \pm 0,96)\end{array}$ & $\begin{array}{c}17,71 \\
( \pm 1,80)\end{array}$ & $14,78( \pm 2,44)$ & 122 & $\begin{array}{c}11 \\
( \pm 1,13)\end{array}$ & $\begin{array}{c}20,05 \\
( \pm 2,27)\end{array}$ & $\begin{array}{c}16,29 \\
( \pm 1,44)\end{array}$ \\
\hline M10 & 107 & $\begin{array}{c}9,92 \\
( \pm 0,51)\end{array}$ & $\begin{array}{c}17,89 \\
( \pm 0,93)\end{array}$ & $14,56( \pm 0,73)$ & 94 & $\begin{array}{c}12,14 \\
( \pm 1,77)\end{array}$ & $\begin{array}{c}21,44 \\
( \pm 2,70)\end{array}$ & $\begin{array}{c}17,13 \\
( \pm 2,10)\end{array}$ \\
\hline M11 & 37 & $\begin{array}{c}9,25 \\
( \pm 1,5)\end{array}$ & $\begin{array}{c}17,5 \\
( \pm 1,29)\end{array}$ & $\begin{array}{c}13 \\
( \pm 1,58)\end{array}$ & 111 & $\begin{array}{c}12,31 \\
( \pm 1,44)\end{array}$ & $\begin{array}{c}20,75 \\
( \pm 1,83)\end{array}$ & $\begin{array}{c}18,63 \\
( \pm 2,39)\end{array}$ \\
\hline
\end{tabular}

Nota: M: Marisqueiras; N: Total de amostras; AC: Altura da Concha (mm); LC: Largura da Concha (mm); CC: Comprimento da Concha (mm). 
Barreira e Araújo (2005) trazem que a diferenciação sexual, bem como o período de maturação do marisco, inicia a partir dos $12,9 \mathrm{~mm}$ para o comprimento da concha, o que corrobora com a medida utilizada pelas marisqueiras da RESEX acerca da catação apenas de tamanhos graúdos. Isso implica que, quando ocorre a pesca com preferência pelos tamanhos maiores, os mariscos coletados já estão maduros sexualmente, ou seja, já iniciaram o seu ciclo reprodutivo. Logo, a ação de separação dos tamanhos tem implicações etnoconservacionistas, que garantem a pesca sustentável, realizada consciente ou inconscientemente, quando essa atitude é referente à questão do rendimento da carne. O rendimento da carne é calculado a partir da relação entre o peso da biomassa e o peso total.

Além de coincidir com o período de maturação da espécie, essa atitude condiz com o proposto pelo acordo de gestão da RESEX, que é de $15 \mathrm{~mm}$ para a CC. Mesmo assim, é necessário maior divulgação dessa normativa nas comunidades beneficiárias, para que todos tomem consentimento, incentivando, de certa forma, a pesca sustentável. Outra RESEX que apresenta uma portaria ( $\mathrm{n}^{\mathrm{o}}$ 187, de 2013), que aborda as normas para uso do marisco, é a RESEX de Pirajubaé em Florianópolis, trazendo como tamanho mínimo para a pesca o de $20 \mathrm{~mm}$, superior ao estabelecido na RESEX Acaú-Goiana, e o encontrado no presente estudo. Esse fato pode estar ligado a uma melhor condição das populações de marisco no local.

Dessa forma, o descarte dos tamanhos juvenis apresenta relevante importância, visto que esses, como ainda não estão maduros, não reproduziram, mas vão ter a oportunidade de completar o seu ciclo de vida, garantindo a continuidade da espécie e agregando, assim, a preservação de toda a arte pesqueira. Quando o processo de pesca influencia na disposição de indivíduos aptos para a reprodução, pode-se acontecer o evento chamado de sobrepesca, influenciando nas gerações futuras e podendo acarretar mudanças na dinâmica populacional do marisco (Bandeira et al., 2017).

Em estudo realizado na Área de Proteção (APA) Barra do Rio Mamanguape, na Paraíba, percebeu-se que a atividade de catação do marisco afeta a disposição dos tamanhos morfométricos da concha, porém, por si só não afeta diretamente na redução do tamanho médio desse bivalve (Cidreira-Neto et al., 2018). Dessa forma, outros fatores maiores, como a poluição, podem ser o agente que influencia na redução da quantidade de mariscos. Estudo realizado com os mariscos no rio Paraíba do Norte, em Bayeux, na Paraíba, demonstra que nas croas de coleta mais próximas a centros urbanos, expostas a maiores gradientes de poluição, o tamanho médio dos mariscos é inferior, bem como uma menor quantidade de bactérias endógenas (Nascimento et al., 2018).

$\mathrm{Na}$ área central da RESEX existem viveiros de carcinicultura com elevado potencial de produção e distribuição de camarões, que são anteriores à criação da UC. Essa atividade influencia na dinâmica populacional de mariscos e peixes, afetando na diminuição das populações, devido à liberação dos dejetos nos rios, afetando as populações que tiram o sustento de forma artesanal desses locais (Silva \& Almeida, 2016). Além dos conflitos sociais causados entre pescadores e produtores de camarão, existe a questão ambiental, visto que a atividade vem como um agente que agrava a qualidade do estuário. Segundo as pescadoras, existe denúncia de que os seguranças dos viveiros estão cortando redes dos pescadores, andando armados e ameaçando os pescadores: "o rio não é deles, é do pescador".

Por diversas vezes o conhecimento tradicional e o conhecimento científico se intercruzam, servindo para comprovar e aprovar saberes e técnicas realizadas de formas artesanais que servem de exemplo para a conservação ambiental, como no caso da separação dos tamanhos de marisco. Palheta, Canete e Cardoso (2016, p. 616), a partir de um estudo 
realizado com as mulheres pescadoras da RESEX Marinha Mãe Grande de Curuçá, acerca da participação feminina quanto pescadora, realizaram uma importante reflexão:

Esse conhecimento tradicional é de fundamental importância no setor pesqueiro e, como visto, contribui para que haja novas possibilidades de uso dos recursos naturais, embora, perante a sociedade, a atividade pesqueira exercida pelas mulheres não seja reconhecida como deveria ser, pois muitas vezes são consideradas apenas ajudante de seus maridos.

Dessa forma, fica perceptível que essa pequena atitude interiorizada por muitas pescadoras marisqueiras pode servir como base para a implementação de políticas pesqueiras, garantindo a perpetuação da tradicionalidade e conservação ambiental.

A prática de coleta apenas dos tamanhos graúdos é algo que necessita de incentivo, visto que respeita o período de maturação sexual dos mariscos, garantindo a permanência da espécie no local, benéfico para o meio ambiente e para as próprias marisqueiras. Quando se trata de unidades de conservação, a temática necessita de mais atenção, servindo de base para a implementação de políticas, como acordo de gestão e plano de manejo, que garantam uma pesca ética e artesanal.

Agradecimentos - Agradecemos a todos os comunitários da RESEX Acaú-Goiana, por darem todo o apoio no desenvolvimento da pesquisa, assim como à Fundação de Amparo à Ciência e Tecnologia do Estado de Pernambuco (Facepe) (IBPG - 1179-9.25/16).

\section{REFERÊNCIAS BIBLIOGRÁFICAS}

Bandeira, F.O.; Camargo, M.; Ramos, J.A.A.; Estupinan, R. \& Santos, A.P.S. Parâmetros biométricos de Anomalocardia flexuosa (Linnaeus, 1767) - Bivalvia - Veneridae no estuário do Rio Paraíba - PB. Gaia Scientia, v. 11, n. 2, p. 242-249, 2017.

Barbosa, J.A.A. \& Aguiar, J.O. Etnoconservação e história ambiental para um novo modelo conservacionista do século XXI. Novos Cadernos NAEA, v. 21, n. 1, p. 243-255, 2018.

Barreira, C.A.R. \& Araújo, M.L.R. Ciclo reprodutivo de Anomalocardia brasiliana (Gmelin, 1791) (Mollusca, Bivalvia, Veneridae) na praia do Canto da Barra, Fortim, Ceará, Brasil. Boletim do Instituto de Pesca, v. 31, n. 1, p. 9-20, 2005.

Berkes, F. Sacred ecology: traditional ecological knowledge and management systems. Taylor \& Francis, 1999.

Bezerril, G. Trabalho no mangue: os saberes e a busca por valorização das marisqueiras de Fortim - Ceará. Cadernos do LEME, v. 4, n. 1, p. 5-33, 2012.

Caetano, H.S. Da ocupação do território: práticas e interações entre marisqueiras no ambiente pesqueiro. Revista de Gestão e Sustentabilidade Ambiental, v. 3, n. 2, p. 204-222, 2015.

Cidreira-Neto, I.R.G.; Nascimento, D.M.; Moraes, P.X. \& Rodrigues, G.G. Análise biométrica de Anomalocardia flexuosa em área de proteção ambiental. Journal Environmental Analysis and Progress, v. 3, n. 2, p. 191-199, 2018.

Dias, T.L.P.; Rosa, R.S. \& Damasceno, L.C.P. Aspectos socioeconômicos, percepção ambiental e perspectivas das mulheres marisqueiras da Reserva de Desenvolvimento Sustentável Ponta do Tubarão (Rio Grande do Norte, Brasil). Gaia Scientia, v. 1, n. 1, p. 25-35, 2007. 
Diegues, A.C.S. Pescadores, camponeses e trabalhadores do mar. São Paulo: Ática, 1983.

Diegues, A.C. Etnoconservação: novos rumos para a proteção da natureza nos trópicos. 2000.

Gil, A.C. Métodos e técnicas de pesquisa social. Editora Atlas: 2008.

Lima, M.E.A.; Selva, V.S.F. \& Rodrigues, G.G. Gestão participativa nas reservas extrativistas: a atuação do Instituto Chico Mendes da Biodiversidade. Revista Brasileira de Geografia Física, v. 9, n. 4, p. 1072-1087, 2016.

Lopéz-Rocha, J.A.; Melo, F.J.F.R.; Gastélum-Nava, E.; Larios-Castro, E. \& Romo-Piñera, A. Morphometric relationship, growth parameters, and natural mortality as estimated primary inputs for fishery management in newfishing areas for bivalve molluscs (BIVALVIA: VENERIDAE). Journal os Shellfish Research, v. 37, n. 3, p. 591-600, 2018.

Nascimento, C.H.V.; Cidreira-Neto, I.R.G.; Silva, R.P.; Assis, J.E.; Gusmão, N.B. \& Rodrigues, G.G. Caracterização morfométrica e microbiota endógena de populações do marisco Anomalocardia flexuasa Linnaeus, 1767 (Bivalvia: Veneridae). Journal Environmental Analysis and Progress, v. 3, n. 3, p. 275-286, 2018.

Nishida, A.K.; Nordi, N. \& Alves, R.R.N. Abordagem etnoecológica da coleta de moluscos no litoral paraibano. Tropical Oceanography, v. 32, n. 1, p. 53-68, 2004.

Palheta, M.K.S.; Canete, V.R. \& Cardoso, D.M. Mulher e mercado: participação e conhecimentos femininos na inserção de novas espécies de pescado no mercado e na dieta alimentar dos pescadores da RESEX Mãe Grande em Curuçá (PA). Boletim do Museu Paraense Emílio Goeldi, v. 11, n. 3, p. 601-619, 2016.

Pereira, B.E. \& Diegues, A.C. Conhecimento de populações tradicionais como possibilidade de conservação da natureza: uma reflexão sobre a perspectiva da etnoconservação. Revista de Desenvolvimento e Meio Ambiente, n. 22, p. 37-50, 2010.

Silva, M.S.F. \& Almeida, G.L. O uso do potencial fitogeográfico pelas comunidades tradicionais em Indiaroba-SE. REGNE, v. 2, 2016.

Silva, J.S. \& Martins, I.X. A pesca de moluscos em ambientes intermareais no oeste do Estado do Rio Grande do Norte, Brasil. Arq. de Ciên. do Mar, v. 50, n. 2, p. 110-118, 2017.

Souto, F.J.B. \& Martins, V.S. Conhecimentos etnoecológicos na mariscagem de moluscos bivalves no manguezal do distrito de Acupe, Santo Amaro - BA. Biotemas, v. 22, n. 4, p. 207-208, 2009. 